\title{
When a new definition overhauls perceptions of MAFLD related cirrhosis care
}

\author{
Kenneth I. Zheng', Mohammed Eslam², Jacob George ${ }^{1}$, Ming-Hua Zheng ${ }^{1,3,4}$ \\ ${ }^{1}$ MAFLD Research Center, Department of Hepatology, the First Affiliated Hospital of Wenzhou Medical University, Wenzhou, China; ${ }^{2}$ Storr \\ Liver Centre, Westmead Institute for Medical Research, Westmead Hospital and University of Sydney, Sydney, Australia; ${ }^{3}$ Institute of Hepatology, \\ Wenzhou Medical University, Wenzhou, China; ${ }^{4}$ Key Laboratory of Diagnosis and Treatment for The Development of Chronic Liver Disease in \\ Zhejiang Province, Wenzhou, China \\ Correspondence to: Ming-Hua Zheng, MD, PhD. MAFLD Research Center, Department of Hepatology, the First Affiliated Hospital of \\ Wenzhou Medical University, No. 2 Fuxue Lane, Wenzhou 325000, China. Email: zhengmh@wmu.edu.cn; Mohammed Eslam, MD, PhD. \\ Storr Liver Centre, Westmead Institute for Medical Research, Westmead Hospital and University of Sydney, Westmead 2145, NSW, Australia. \\ Email: mohammed.eslam@sydney.edu.au; Jacob George, MD, PhD. Storr Liver Centre, Westmead Institute for Medical Research, Westmead \\ Hospital and University of Sydney, Westmead 2145, NSW, Australia. Email: jacob.george@sydney.edu.au.
}

Submitted Sep 23, 2020. Accepted for publication Oct 09, 2020.

doi: $10.21037 / \mathrm{hbsn}-20-725$

View this article at: http://dx.doi.org/10.21037/hbsn-20-725

Metabolic-associated fatty liver disease (MAFLD) (1), formerly known as non-alcoholic fatty liver disease, is a major causes of liver cirrhosis (2). MAFLD represents a growing global health and economic burden since approximately one in four people have the disease (3). As a consequence, MAFLD-related cirrhosis is expected to become the leading cause for liver failure and liver cancer, and the major indication for liver transplantation this century (3).

\section{Improvement in diagnosis}

The diagnosis of MAFLD cirrhosis poses unique challenges for clinicians. Essentially, two patient groups are possible, one who meet the current criteria for MAFLD and the second, a group that has the clinical profile of MAFLD but without significant hepatic steatosis $(<5 \%)$. The latter group by current definitions, is termed cryptogenic cirrhosis. Most clinicians believe that the majority of these patients represent MAFLD in which hepatic steatosis is no longer present. Indeed, a plausible and cogent explanation for the phenotype exists with van der Poorten et al. (4), demonstrating that an intimate relationship between adiponectin levels, bile acids, and adipocyte fexaramine activation in vivo and in vitro results in hepatic fat loss in cirrhosis from MAFLD. While there remains debate as to whether all cryptogenic cirrhosis is a consequence of MAFLD, Thuluvath et al. (5), demonstrated that cryptogenic cirrhosis at least as listed in the UNOS database, likely represents cirrhosis related to MAFLD as well as a range of other previously undiagnosed conditions (e.g., autoimmune hepatitis, alcohol associated liver disease etc.). The sum of this data suggests that a clearer definition is needed to unequivocally define cirrhosis resulting from MAFLD. The newly proposed diagnostic criteria for MAFLD cirrhosis by Eslam et al. (6), includes past or present evidence of metabolic risk factors for MAFLD in addition to either evidence of MAFLD on prior liver biopsy or historical documentation of steatosis by hepatic imaging (or other means). With these positive criteria, patients previously diagnosed with cryptogenic cirrhosis (due to lack of histological features of steatosis) may now be identified correctly as MAFLD cirrhosis. Unfortunately, because of lack of prior awareness of the prevalence of MAFLD by both patients and practitioners alike, there will remain a subset diagnosed with cirrhosis at presentation and no liver fat, whose liver disease is most likely a consequence of MAFLD, but in whom other etiologies such as alcohol and autoimmune disease cannot be excluded. Cryptogenic cirrhosis will remain the most appropriate term for these individuals. It is worth noting that the proposed criteria do not preclude MAFLD cirrhosis patients from having 
a contribution to their cirrhosis for alcohol, autoimmune disease, etc. (i.e., two or more etiologies is possible and even likely). In this context, the new definition provides a conceptual framework for categorization. With time and increasing awareness of MAFLD, we expect that there will be a shift to fewer cases of so-called cryptogenic cirrhosis as MAFLD is diagnosed using the positive criteria proposed, prior to the development of cirrhosis.

\section{Management of MAFLD-cirrhosis}

There is no one-size-fits-all approach for managing liver cirrhosis; this is also true for MAFLD related cirrhosis. The major focus of treatment for MAFLD cirrhosis comprises management of complications related to decompensation and hepatocellular carcinoma (HCC) surveillance. However, this reactionary approach often places a substantial burden on the healthcare system. Aside from treating decompensation events, cirrhosis management should focus on education, lifestyle modification, protecting the liver from further injury (e.g., through vaccination for viral hepatitis and avoidance of hepatotoxic medications), and care coordination (2).

The liver has considerable regenerative potential, and cirrhosis regression and "re-compensation" have been described in patients with alcoholic cirrhosis following abstention from alcohol, cure of hepatitis $\mathrm{C}$ or suppression of hepatitis B, and as well in the context of steatohepatitis, after bariatric surgery (2). To slow disease progression in MAFLD cirrhosis, diet and exercise can be an effective intervention, but it remains critical to avoid sarcopenia. In addition, sarcopenia is an adverse prognostic factor in patients with HCC (7). While physical activity is important, meticulous attention to body composition that includes body fat and skeletal muscle mass is needed when managing patients with MAFLD cirrhosis.

Appropriate surveillance for HCC and gastroesophageal varices is recommended because it improves overall survival. HCC screening in these patients is achieved by utilizing imaging modalities and measuring serum alpha-fetoprotein every 6 months (8). It should be noted however that the sensitivity and specificity of ultrasound-based surveillance in cirrhosis is suboptimal, while obesity can further impact good hepatic visualization. It was recently demonstrated that in 224 Chinese patients with MAFLD related compensated cirrhosis, Baveno VI Criteria (9) can be used as a risk stratification tool for the presence of high-risk varices (10).
However, the time interval for variceal screening remains uncertain because obesity worsens the progression of cirrhosis from any etiology; thus, more frequent screening might be indicated in high risk patients (9).

\section{Precision care for MAFLD-cirrhosis}

Care for MAFLD cirrhosis patients is complex. It is noteworthy that a recent report from 29 European countries (Norway, Switzerland and all European Union countries expect for Malta) reported that none had written strategies or action plans for this disease (11). As a result, physicians (non-hepatologists) are not likely to recognize MAFLD cirrhosis and to subsequently administer the most appropriate care. From 2013 to 2015, three hospitals in the UK implemented a 'care bundle' to ensure evidence-based treatments are delivered to cirrhosis patients within $24 \mathrm{~h}$ of hospital admission (12). The care bundle allowed physicians (non-specialists) to follow a simple checklist of important initial investigations, and provided step-by-step guidance for managing infections, spontaneous bacterial peritonitis, gastrointestinal bleeding and acute kidney injury. Though the process often required repeated education and reinforcement, Dyson et al. (12) reported major improvements in the delivery of indicated care (i.e., increased rates of endoscopic screening, ascitic taps, administration of terlipressin and prophylactic antibiotics) following introduction of the care bundle. The success of the 'care bundle' provides a new vista for ways to manage MAFLD cirrhosis from diagnosis to appropriate care (see Figure 1).

Outside of hospital settings, MAFLD patients will likely receive enormous benefits from chronic disease management (CDM) (13), a concept for optimizing care between hospital visits to improve disease outcomes. CMD protocolizes medical care delivery, self-management, and community support. For example, prevention of gastroesophageal bleeding is more likely to be achieved with a system in place for personalized alerts for periodic surveillance. Similarly, lifestyle intervention is best accomplished by self-management where patients can benefit from continuous feedback on their weight loss goals and activity levels. Community support also involves a patient's social networks (e.g., family, friends, caregiver) and has considerable impact on their ability to manage their disease. Moreover, healthcare provider and nurseled management can ensure and aid the implementation of highly individualized CDM protocols. 






Figure 1 Summary of non-specific (A) and MAFLD-specific (B) care elements for managing MAFLD related cirrhosis.

\section{Future of MAFLD-cirrhosis care}

The new diagnosis criteria have great potential to improve the care of MAFLD patients with cirrhosis. With a clear diagnosis, innovative biometrics may be investigated to properly identify the predictors of decompensation events and risk of future HCC. Further understanding of metabolic dysfunction among MAFLD patients will permit the best refinement of individualised predictions of outcomes. Many hepatologists are already experimenting with multi-disciplinary approaches (involving nutrition, diabetology, cardiology, renal, etc.) in their management of MAFLD patients. This promotes the integration of multidisciplinary approaches to CDM in a scientifically rigorous manner. Thus, we encourage colleagues to conduct studies to help shape the future of MAFLD-specific cirrhosis care and to come together as a medical community to share knowledge of existing care models and how to best translate them into the practice setting.

Currently, there is very little knowledge and data on how to better treat patients with MAFLD cirrhosis. Even if there were, we need to have mechanisms in place to evaluate the efficacy of management. Assessing the quality of care, especially in the absence of an established algorithm tailored specifically for these patients is problematic. Kanwal et al. (14), recently reported a gap between care indicated and care delivered for cirrhotic patients by evaluating eight quality indicators that they developed. Such methodology can provide a framework for evaluating the current and future management of MAFLD cirrhosis. The application of personalized care for MAFLD cirrhosis patients can be expected to yield two benefits: promotion of care quality by improving outcomes and an increase in return-on-healthcare-investment to alleviate the economic burden of MAFLD. However, to achieve this, adequate resources need to be allocated for developing, establishing and implementing an effective and precise algorithm for the management of MAFLD cirrhosis in and outside of the hospital setting.

\section{Acknowledgments}

Funding: ME and JG are supported by the Robert W. Storr Bequest to the Sydney Medical Foundation, University of Sydney; a National Health and Medical Research Council of Australia (NHMRC) Program Grant (APP1053206, APP1149976) and Project grants (APP1107178 and APP1108422). MHZ is supported by grants from the National Natural Science Foundation of China (82070588), High Level Creative Talents from Department of Public Health in Zhejiang Province (S2032102600032) and Project of New Century 551 Talent Nurturing in Wenzhou. 


\section{Footnote}

Provenance and Peer Review: This article was commissioned by the editorial office, Hepatobiliary Surgery and Nutrition. The article did not undergo external peer review.

Conflicts of Interest: All authors have completed the ICMJE uniform disclosure form (available at http://dx.doi. org/10.21037/hbsn-20-725). The authors have no conflicts of interest to declare.

Ethical Statement: The authors are accountable for all aspects of the work in ensuring that questions related to the accuracy or integrity of any part of the work are appropriately investigated and resolved.

Open Access Statement: This is an Open Access article distributed in accordance with the Creative Commons Attribution-NonCommercial-NoDerivs 4.0 International License (CC BY-NC-ND 4.0), which permits the noncommercial replication and distribution of the article with the strict proviso that no changes or edits are made and the original work is properly cited (including links to both the formal publication through the relevant DOI and the license). See: https://creativecommons.org/licenses/by-nc-nd/4.0/.

\section{References}

1. Eslam M, Sanyal AJ, George J. MAFLD: A ConsensusDriven Proposed Nomenclature for Metabolic Associated Fatty Liver Disease. Gastroenterology 2020;158:19992014.e1.

2. Ge PS, Runyon BA. Treatment of Patients with Cirrhosis. N Engl J Med 2016;375:767-77.

3. Estes C, Razavi H, Loomba R, et al. Modeling the epidemic of nonalcoholic fatty liver disease demonstrates an exponential increase in burden of disease. Hepatology 2018;67:123-33.

4. van der Poorten D, Samer CF, Ramezani-Moghadam $\mathrm{M}$, et al. Hepatic fat loss in advanced nonalcoholic

Cite this article as: Zheng KI, Eslam M, George J, Zheng MH. When a new definition overhauls perceptions of MAFLD related cirrhosis care. HepatoBiliary Surg Nutr 2020;9(6):801804. doi: 10.21037/hbsn-20-725 steatohepatitis: are alterations in serum adiponectin the cause? Hepatology 2013;57:2180-8.

5. Thuluvath PJ, Kantsevoy S, Thuluvath AJ, et al. Is cryptogenic cirrhosis different from NASH cirrhosis? J Hepatol 2018;68:519-25.

6. Eslam M, Newsome PN, Sarin SK, et al. A new definition for metabolic dysfunction-associated fatty liver disease: An international expert consensus statement. J Hepatol 2020;73:202-9.

7. Fujiwara N, Nakagawa H, Kudo Y, et al. Sarcopenia, intramuscular fat deposition, and visceral adiposity independently predict the outcomes of hepatocellular carcinoma. J Hepatol 2015;63:131-40.

8. Eslam M, Sarin SK, Wong VWS, et al. The Asian Pacific Association for the Study of the Liver clinical practice guidelines for the diagnosis and management of metabolic associated fatty liver diseases. Hepatol Int 2020. [Epub ahead of print].

9. de Franchis R. Expanding consensus in portal hypertension: Report of the Baveno VI Consensus Workshop: Stratifying risk and individualizing care for portal hypertension. J Hepatol 2015;63:743-52.

10. Zheng KI, Liu C, Li J, et al. Validation of Baveno VI and Expanded Baveno VI criteria to identify high-risk varices in patients with MAFLD-related compensated cirrhosis. J Hepatol 2020;73:1571-3.

11. Lazarus JV, Ekstedt M, Marchesini G, et al. A crosssectional study of the public health response to nonalcoholic fatty liver disease in Europe. J Hepatol 2020;72:14-24.

12. Dyson JK, Rajasekhar P, Wetten A, et al. Implementation of a 'care bundle' improves the management of patients admitted to hospital with decompensated cirrhosis. Aliment Pharmacol Ther 2016;44:1030-8.

13. Volk ML, Piette JD, Singal AS, et al. Chronic disease management for patients with cirrhosis. Gastroenterology 2010;139:14-6.e1.

14. Kanwal F, Kramer J, Asch SM, et al. An explicit quality indicator set for measurement of quality of care in patients with cirrhosis. Clin Gastroenterol Hepatol 2010;8:709-17. 